\title{
Descriptive Study of Exposure to Inhalation Zanamivir and Pregnancy-Related Outcomes
}

\author{
Walker $\mathrm{AM}^{* 1}$, Vannappagari $\mathrm{V}^{2,3}$, Buus $\mathrm{R}^{4}$, Covington $\mathrm{D}^{4}$, Bratton $\mathrm{E}^{2}$ and Thompson $\mathrm{M}^{5}$ \\ ${ }^{1}$ WHISCON, Newton, MA, US \\ ${ }^{2}$ Glaxo Smith Kline, Research Triangle Park, NC, US \\ ${ }^{3}$ Gillings School of Global Public Health, University of North Carolina-Chapel Hill, NC, United States of America \\ ${ }^{4} \mathrm{PPD}$, Inc., Wilmington, NC, US \\ ${ }^{5}$ Cegedim Strategic Data Medical Research, Ltd., London, UK
}

\section{${ }^{*}$ Corresponding author: Walker AM, WHISCON, Newton, MA, US E-mail: alec.walker@whiscon.com}

Citation: Walker AM, Vannappagari V, Buus R, Covington D, Bratton E, et al. (2014) Descriptive Study of Exposure to Inhalation Zanamivir and Pregnancy-Related Outcomes. J Pharm Drug Devel 2(3): 302. doi: 10.15744/2348-9782.1.502

Received Date: May 23, 2014 Accepted Date: October 21, 2014 Published Date: October 23, 2014

\begin{abstract}
Purpose: To assess the effect of neuraminidase inhibitor (NI), zanamivir, on pregnancy outcomes.

Methods: The Health Improvement Network (THIN) provided data from UK General Practitioners (GPs) for 144 pregnant women who were prescribed zanamivir and 144 age- and date-matched untreated comparators with no recorded diagnosis of influenza. Groups were assessed for baseline characteristics, treatment-emergent diagnoses in the mother, pregnancy outcomes and congenital malformations diagnosed in the offspring within 28 days of birth.

Results: Treatment and comparator groups were similar at baseline. Most (100/144) zanamivir prescriptions occurred during the second and third trimesters. Groups were similar in recorded smoking during pregnancy; more women in the comparator group had recorded alcohol use. The zanamivir group had more diagnoses compatible with influenza-like illness (ILI) and was more often prescribed antibiotics, analgesics and medicines for respiratory and gastrointestinal symptoms. Pregnancy outcomes other than live birth were not found in the GP record, possibly due in part to cohort entry having occurred late in most pregnancies. There was no difference between groups in the risk of any treatment-emergent diagnosis. Congenital anomalies occurred in similar proportions, but infants born to zanamivir-exposed women had substantially fewer diagnoses from the cluster of cardiac defects consisting of atrial and ventricular septal defects and patent ductus arteriosus (1 zanamivir, 6 comparators).

Conclusions: Zanamivir-exposed pregnant women have a wide range of symptoms of and treatments for ILI that complicate comparisons with untreated women. There was no indication of increased risk to the mother, the pregnancy or the infant following exposure.

Keywords: Influenza; Pregnancy; Drug safety; Zanamivir; Congenital anomalies

Abbreviations: CDC-The Centers for Disease Control and Prevention; CSD MR-Cegedim Strategic Data Medical Research; EMA-The European Medicines Agency; EUROCAT-European Surveillance of Congenital Anomalies; GP-General practitioner; ICU-Intensive care unit; ILI-Influenza-like illness; LMP-Last menstrual period; NI-Neuraminidase Inhibitor; UK-United Kingdom; US-United States
\end{abstract}

\section{Introduction}

Influenza is the most common cause of serious respiratory illness worldwide [1]. In the United States, influenza causes approximately 36,000 deaths and 226,000 hospitalizations in an average season [2]. The $2009 \mathrm{H} 1 \mathrm{~N} 1$ influenza pandemic led to greater morbidity and mortality, particularly for those aged less than 65 years. Pandemic influenza affected over 214 countries in 2009 and caused over 18,500 laboratory-confirmed deaths. Globally, there were an estimated 201,200 respiratory deaths (range: 105,700-395,600) with an additional 83,300 cardiovascular deaths (range: 46,000-179,900) associated with 2009 pandemic influenza A H1N1 [3].

McLean and Pebody (2010) estimated a total of 784,000 (range: 372,000 - 1,638,000) clinical cases of influenza-like illness (ILI) due to pandemic H1N1 2009 in England [4]. From the beginning of the pandemic to April 2010, 2,831 patients were hospitalized with confirmed pandemic H1N1 2009 infection. While pregnant women were not at increased risk of influenza infection, once infected, they were five times more likely to be hospitalized (RR 5.1; 95\%CI $4.2-6.1$ ) and seven times more likely to die, in comparison to healthy females of child-bearing age (RR: 7; 95\% CI: 3-15) [4,5]. Ten of the 361 reported H1N1 deaths with available risk information were among pregnant women [4]. 
Although there is strong evidence that influenza infection endangers the pregnant woman and her pregnancy, there is little information about the safety of zanamivir in pregnant women. The primary objective of this study has been to evaluate the safety of zanamivir in pregnancy, focusing on birth outcomes, congenital anomalies identifiable at or shortly after birth and treatmentemergent diagnoses in the mother. Secondary objectives were to identify the characteristics of zanamivir recipients at the time of treatment and to characterize the clinical circumstances under which zanamivir was prescribed.

\section{H1N1 Influenza and Pregnancy}

Pregnant women with influenza exhibit more severe symptoms and experience higher mortality than do women of the same age in the general population [6]. For pandemic H1N1, the risk of intensive care unit (ICU) admission or death was 7.0 per 100,000 for pregnant women versus 1.7 per 100,000 for non-pregnant women of reproductive age [7]. Creanga et al. (2011) reported that ICU admissions were more than twice as common in 2009 in pregnant women with H1N1 than among pregnant women during seasonal influenza (11.7\% vs. 5.3\%) [6]. The Centers for Disease Control and Prevention (CDC) in the USA found 30 (5\%) of 593 reported H1N1 deaths through August 2009 were in pregnant women, who account for only about $1 \%$ of the general population [8].

Influenza infection can result in pregnancy complications, particularly in later stages of pregnancy and in women with comorbid conditions. In a recent systematic review of 120 publications of the effects of H1N1 infection on 3,110 pregnant women from 29 countries, Mosby et al (2011) reported that almost one third of pregnant women with reported influenza had risk factors or unspecified co-morbidities for severe disease other than pregnancy [9]. Almost 50\% of pregnant women with severe influenza illness reported to the CDC from April 15, 2009 to August 10, 2010 had significant underlying comorbidity. Among these, women who died had an underlying condition more often than did survivors (62\% vs. 47\%; p=0.04) [10]. Pregnant women hospitalized with confirmed $\mathrm{H} 1 \mathrm{~N} 1$ in the UK were more likely to have suffered from asthma that required inhaled or oral steroids or have been obese than comparison women [11].

\section{H1N1 Influenza and Birth Outcomes}

The fetal effects of influenza infection include increases in risk of stillbirth, spontaneous abortion, preterm birth and certain congenital abnormalities [12]. High fever rather than influenza per se may be the cause of neural tube defects and other abnormalities $[13,14]$.

Infants born to H1N1-infected women generally do well, but are more likely to be born prematurely. Compared to a baseline rate of $9.6 \%$ worldwide [15], the rate of preterm birth among H1N1-infected women was 15\% on the French island of La Reunion and $\geq 30 \%$ in the UK, Australia, and the US [9]. In the UK, Yates et al. (2010) found that pregnant women admitted to a hospital with $\mathrm{H} 1 \mathrm{~N} 1$ infection had at least three times the odds of delivering preterm compared to uninfected women $(\mathrm{OR}=3.1,95 \% \mathrm{CI}$ : $2.1-4.5)$ [11]. In the US many newborns from influenza-affected pregnancies required hospitalization and ICU admission [9]. Among 85 infants born during maternal hospitalization for influenza, 64\% were preterm compared with $12 \%$ in the general US population and $44 \%$ were low birth weight compared with $8 \%$ nationwide [10]. Among 54 infants born after maternal hospitalization for influenza, 25\% were small for gestational age compared with $10 \%$ nationally [10]. Those hospitalized pregnant women also eventually experienced 11 spontaneous abortions, seven fetal deaths and one ectopic pregnancy. One had an elected abortion. A number of other studies from the US, UK, Australia and Israel have reported stillbirths, neonatal deaths and postnatal deaths. The numbers involved are small and it is not always clear whether death in particular was due to H1N1 infection [8,16-20].

Compared to the baseline cesarean delivery rate of $31 \%$ in the US, Siston et al. (2010) reported that 58\% of pregnant women with $\mathrm{H} 1 \mathrm{~N} 1$ in the US delivered by cesarean section [8], with the procedure commonly carried out for maternal hypoxemia and decompensation [9].

\section{H1N1 Antiviral Treatment for Pregnant Women}

The CDC recommends that pregnant women with suspected influenza receive prompt early empiric treatment with appropriate influenza antiviral medications [21]. Treatment within two days of illness onset reduces the likelihood of ICU admission or death [22].

The NI zanamivir was first authorized in Sweden in 1999 and is indicated for treatment and prevention of influenza in adults and children over the age of 5 years. In pregnant rats and rabbits, zanamivir administration did not result in toxicity to the mother or fetus, and no congenital malformations occurred [23]. Zanamivir crosses the placenta in animal models and is excreted in breast milk of rats [23]. There have been two published reports of adverse events, among the three pregnant women exposed to zanamivir during clinical trials [24]. One pregnancy resulted in elective termination, the second in spontaneous abortion. There were no adverse events noted in the third pregnancy. The Japan Drug Information Institute in Pregnancy reported a healthy term baby was born after zanamivir exposure at four weeks gestation [14]. In a matched observational cohort study of 86 exposed and 860 unexposed pregnancies in Sweden, Svensson et al. (2011) found that infants whose mothers had received oseltamivir or zanamivir during pregnancy had an increased risk of late transient hypoglycemia, but had no effect on several birth outcomes (preterm birth, low birth weight, small-for-gestational age, low Apgar score, congenital malformations, and death) [25]. The European Medicines Agency (EMA) concluded in 2009 that exposure to zanamivir during pregnancy did not represent a safety risk to the fetus [26]. However, clinical experience with use of zanamivir during pregnancy is extremely limited [26]. 


\section{Methods}

This was a retrospective cohort study, based on routinely generated medical records, comparing pregnant women prescribed zanamivir with concurrently pregnant women not prescribed any influenza antiviral agent. Data were collected for pregnancy outcomes or losses between December 2009 and November 2010, with continued infant observation for 28 days after delivery. Study endpoints included pregnancy outcomes, congenital anomalies and treatment-emergent diagnoses in the mother. Results were stratified by trimester of earliest exposure to zanamivir.

\section{Data sources}

CSD MR UK provided access to THIN, which contains electronic medical records of seven million persons from participating general practices in the UK. Information in THIN is limited to data reported to or recorded in primary care, which is encoded using the UK National Health Service's standard Read coding system [27]. Drug exposure comes from the GP's prescribing information. Infant follow-up was possible through linkage with the GP record.

\section{Study population}

Subject pregnancies included those with completion from December 2009 through November 2010 and zanamivir prescription (or the absence of any antiviral exposure in comparators) during the presumed period of pregnancy.

Pregnancy was identified on the basis of a GP record in THIN of last menstrual period (LMP) and, where this was absent, from records of live births in maternal records. Results from these two cohorts were pooled, except for pregnancy outcome, as pregnancies identifiable only through live births by definition do not include those with other outcomes.

One comparator for each woman prescribed zanamivir during pregnancy was selected at random from women meeting the following criteria: born in the same year as the index woman, anticipated date of full-term delivery within 14 days of the corresponding date for the index pregnancy (when pregnancy and LMP were identified in the GP record), and actual date of delivery within 14 days of the corresponding date for the index pregnancy (when pregnancy and LMP were not identified in the GP record). In a preliminary implementation of the protocol, CSD MR UK sought two matching comparison pregnancies from within THIN for each zanamivir-exposed pregnancy and sought to match comparison pregnancies on general practice as well. This proved too restrictive a set of matching criteria to produce two comparators; therefore, 1:1 matching was implemented and the requirement for matching on general practice was dropped.

\section{Data}

The following characteristics of pregnancies were identified from the GP record: trimester of first exposure to zanamivir, maternal comorbidities and maternal medications that were prescribed at least 28 days after the last prescribing of zanamivir or the corresponding date in comparators (determined by the estimated number of days since LMP). Maternal health conditions noted in the GP record within seven days before or after the initial prescription of zanamivir were included in the analysis. All diagnoses that occurred at least 28 days after the date of first zanamivir prescription (or the corresponding date in comparator women) were counted as treatment-emergent if there was no diagnosis sharing the same Read code during the pregnancy up until that time.

The primary study outcome was congenital malformation, as inferred from Read codes assigned to the infant in the first 28 days of life. These were recast into the European Surveillance of Congenital Anomalies (EUROCAT) coding and classification system to yield EUROCAT major and minor anomalies, and other conditions not specifically coded as major or minor anomalies [28]. Congenital malformations in exposed infants were also potential adverse events. These were listed individually with sex, gestational age and trimester of first exposure.

All treatment-emergent diagnoses occurring in zanamivir-exposed women were a secondary outcome. These were also considered as potential adverse events, and line listings for each were obtained to elucidate the timing of concurrent potentially explanatory events in the health record.

Pregnancy outcomes in the GP record were classified as one of the following: elective termination, spontaneous abortion (spontaneous fetal loss at $<20$ weeks' gestation), fetal death (death of a fetus at $\geq 20$ weeks' gestation), premature birth (birth occurring at $<37$ weeks' gestation) and live birth at term. These also formed a secondary outcome.

\section{Ethics Statement}

The protocol for this study was reviewed and approved by an independent Scientific Review Committee (SRC). NCT01462487 is the ClinicalTrials.gov identifier for the protocol summary posting. 
The THIN database is a collection of pseudo-anonymised patient records collected from primary care. No personal information is available in the database. The data collection scheme for THIN is approved by the UK Multicentre Research Ethics Committee (reference number: 07H1102103). The data collection methodology requires the practice to notify the patients, through display material, that medical records in a non-identifiable form are made available for research and giving patients the option to opt out of the data collection scheme. Individual patient consent is not required for database studies.

\section{Results}

\section{Baseline}

We identified 144 pregnancies in which zanamivir was prescribed. Most women (88\%) started treatment in their second trimester (Table 1). Only $56 \%$ had a diagnosis that mentioned influenza within seven days of zanamivir prescription (Table 1). There was one notation each of threatened abortion and one of post coital bleeding. Almost all the other diagnoses identified around the time of first zanamivir dispensing were consistent with an ILI and none appeared to represent illness that might have affected the course or the outcome of pregnancy (data not shown).

\begin{tabular}{|l|c|c|c|c|c|c|c|c|}
\hline \multirow{2}{*}{} & \multicolumn{9}{|c|}{ Trimester } \\
\cline { 2 - 12 } & \multicolumn{2}{|c|}{ First } & \multicolumn{2}{|c|}{ Second } & \multicolumn{2}{|c|}{ Third } & \multicolumn{2}{|c|}{ Total } \\
\cline { 2 - 12 } & 44 & $(\%)$ & 88 & $(\%)$ & 12 & $(\%)$ & 144 & $(\%)$ \\
\hline Flu like illness & 11 & $(25.0)$ & 18 & $(20.4)$ & 1 & $(8.3)$ & 30 & $(20.8)$ \\
\hline Influenza & 9 & $(20.5)$ & 10 & $(11.4)$ & 2 & $(16.7)$ & 21 & $(14.6)$ \\
\hline Influenza-like symptoms & 7 & $(15.9)$ & 6 & $(6.8)$ & & & 13 & $(9.0)$ \\
\hline Influenza A (H1N1) swine influenza & 4 & $(9.1)$ & 2 & $(2.3)$ & 3 & $(25.0)$ & 9 & $(6.3)$ \\
\hline Other specified pneumonia or influenza & 2 & $(4.5)$ & 2 & $(2.3)$ & & & 4 & $(2.8)$ \\
\hline Suspected influenza A virus subtype H1N1 infection & & & 1 & $(1.1)$ & & & 1 & $(<1)$ \\
\hline Suspected swine influenza & & & 1 & $(1.1)$ & & & 1 & $(<1)$ \\
\hline Possible influenza A virus H1N1 subtype & & & & & 1 & $(8.3)$ & 1 & $(<1)$ \\
\hline Influenza like illness & 1 & $(2.3)$ & & & & & 1 & $(<1)$ \\
\hline Total & 34 & $(77.3)$ & 40 & $(45.5)$ & 7 & $(58.3)$ & 81 & $(56.3)$ \\
\hline
\end{tabular}

Table 1: Diagnoses at zanamivir prescription. Diagnoses of influenza assigned \pm 7 days of zanamivir first prescription reported by trimester of treatment initiation among women with a pregnancy outcome between $1^{\text {st }}$ December 2009 and $30^{\text {th }}$ November 2010 .

The most commonly recorded tests in the zanamivir cohort were blood counts and urinalyses, compatible with evaluation of an acute febrile episode, but most tests overall were ones that relate to the management of conditions other than influenza. The Read coding system used in THIN provides a variety of codes for influenza diagnostic tests, including antibody, serology, "virus detected" and nucleic acid detection for a range of influenza types (NHS Information Authority). None of these were documented among the zanamivir-exposed women.

Since zanamivir and comparator cohort sizes were equal, counts could be directly compared. Demographic and baseline characteristics by group and trimester of zanamivir exposure are shown in Table 2 . The age distributions between the study and comparison groups were similar. Information on smoking and alcohol consumption during pregnancy was largely absent from the GP record (47\% and 70\% unknown, respectively, for both zanamivir and comparator groups), and information on use of illicit substances was not available. Among those with available data, 13/77 (17\%) women in the zanamivir group were identified as smokers and 5/43 (12\%) with notes on alcohol were reported to have consumed alcohol. In the comparison group, 12/76 (16\%) women were smokers and 14/42 (33\%) consumed alcohol. The most common morbidities reported prior to cohort entry were heartburn (nine in each group) and hyperemesis gravidarum (six in each group) (Table 2).

Neither the diagnoses assigned nor the drugs prescribed indicate any systematic health differences between the women who received zanamivir and those with the comparator pregnancies. Nor did there appear to be health difference during the pregnancies themselves, other than those that may have been directly associated with the health care episode in which zanamivir was prescribed. While fewer than $10 \%$ of the zanamivir group had any prescribed treatment other than zanamivir, zanamivir recipients had substantially more prescriptions than comparators for antibiotics, analgesics, drugs for respiratory conditions, megaloblastic anemias, and disorders of gastrointestinal motility (Table 3). 


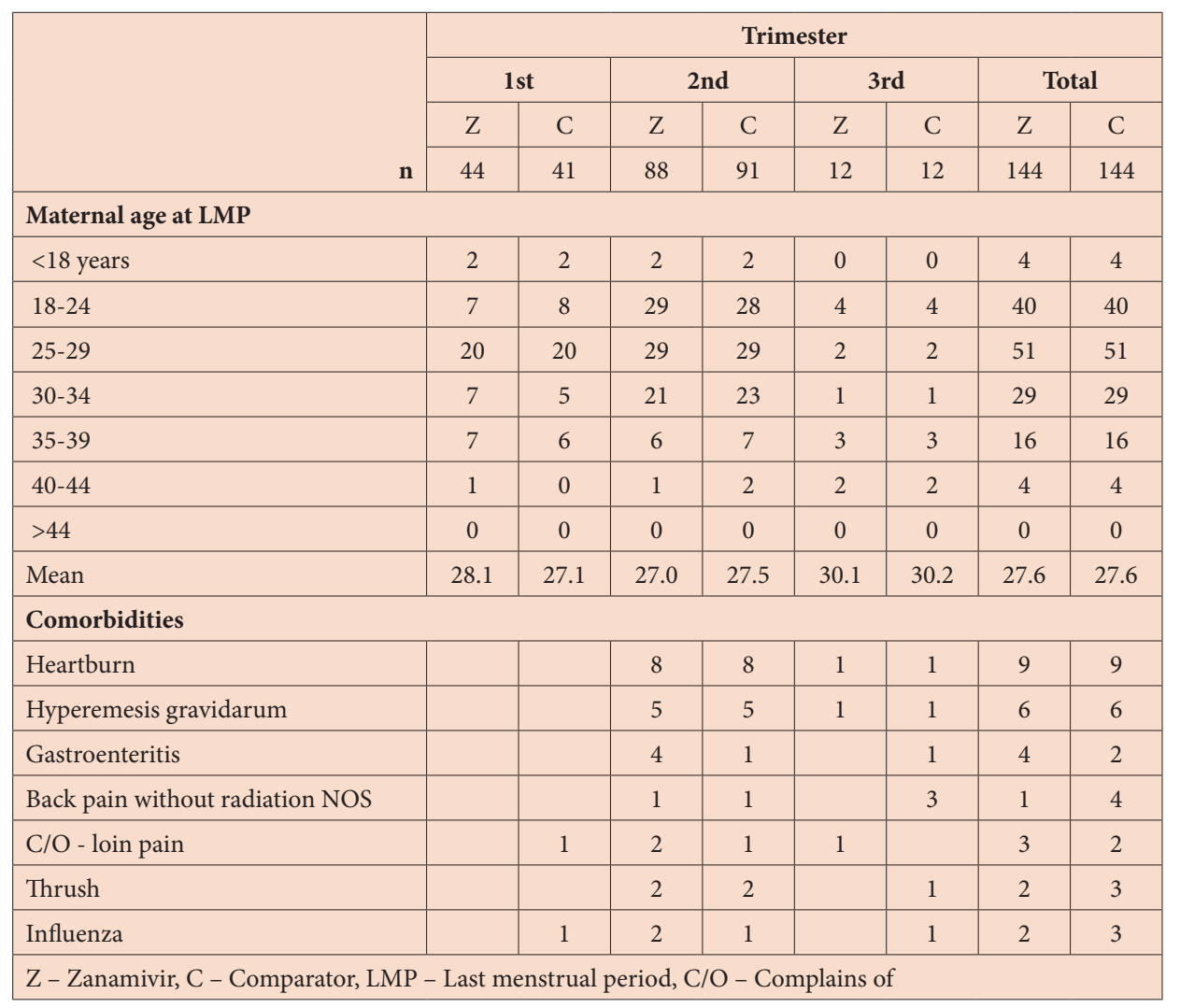

Table 2: Demographics/Baseline characteristics and comorbidities (diagnoses prior to cohort entry date). Maternal characteristics reported by trimester of treatment initiation.

\begin{tabular}{|c|c|c|c|c|c|c|c|c|}
\hline \multirow[b]{3}{*}{ Co-medication } & \multicolumn{6}{|c|}{ Trimester } & & \\
\hline & \multicolumn{2}{|c|}{$\# 1$} & \multicolumn{2}{|c|}{$\# 2$} & \multicolumn{2}{|c|}{$\# 3$} & \multicolumn{2}{|c|}{ Total } \\
\hline & $\mathrm{Z}$ & $\mathrm{C}$ & $\mathrm{Z}$ & $\mathrm{C}$ & $\mathrm{Z}$ & $\mathrm{C}$ & $\mathrm{Z}$ & $\mathrm{C}$ \\
\hline $\mathrm{n}$ & 44 & 41 & 88 & 91 & 12 & 12 & 144 & 144 \\
\hline Influenza (zanamivir) & 44 & & 88 & & 12 & & 144 & 0 \\
\hline Drugs used in megaloblastic anemias & 11 & 7 & 25 & 24 & 4 & 2 & 40 & 33 \\
\hline Broad-spectrum penicillins & 3 & 1 & 15 & 10 & 5 & 1 & 23 & 12 \\
\hline Compound alginates and proprietary indigestion preparations & 4 & & 11 & 8 & 8 & 1 & 23 & 9 \\
\hline Non-opioid analgesics & 3 & 2 & 9 & 7 & 5 & 1 & 17 & 10 \\
\hline Selective beta2 agonists & 3 & & 11 & 6 & 2 & 2 & 16 & 8 \\
\hline Drugs used in nausea and vertigo & 1 & & 14 & 5 & 1 & 1 & 16 & 6 \\
\hline Vaginal and vulval infections & 2 & 1 & 9 & 6 & 2 & 1 & 13 & 8 \\
\hline Oral iron & 4 & & 5 & 7 & 5 & 2 & 14 & 9 \\
\hline Cephalosporins & 3 & 2 & 6 & 7 & 1 & 1 & 10 & 10 \\
\hline Osmotic laxatives & 2 & & 9 & 4 & 1 & 1 & 12 & 5 \\
\hline Corticosteroids & 3 & & 4 & 6 & 3 & 1 & 10 & 7 \\
\hline Topical corticosteroids & 2 & & 10 & 2 & & 3 & 12 & 5 \\
\hline Drugs used in nasal allergy & 2 & & 5 & 6 & 1 & 1 & 8 & 7 \\
\hline Antihistamines & & 2 & 2 & 7 & 1 & 1 & 3 & 10 \\
\hline Antifungal preparations & 2 & & 5 & 4 & 1 & 1 & 8 & 5 \\
\hline Emollients & 2 & & 3 & 1 & 1 & 1 & 6 & 2 \\
\hline Selective serotonin re-uptake inhibitors & 1 & & 2 & 3 & 1 & & 4 & 3 \\
\hline Macrolides & 3 & & 2 & 1 & 1 & & 6 & 1 \\
\hline Other anti-inflammatory preparations & & & 2 & 4 & 1 & & 3 & 4 \\
\hline
\end{tabular}




\begin{tabular}{|c|c|c|c|c|c|c|c|c|}
\hline \multirow[b]{3}{*}{ Co-medication } & \multicolumn{6}{|c|}{ Trimester } & & \\
\hline & \multicolumn{2}{|c|}{$\# 1$} & \multicolumn{2}{|c|}{$\# 2$} & \multicolumn{2}{|c|}{$\# 3$} & \multicolumn{2}{|c|}{ Total } \\
\hline & $\mathrm{Z}$ & $\mathrm{C}$ & $\mathrm{Z}$ & $\mathrm{C}$ & $\mathrm{Z}$ & $\mathrm{C}$ & $\mathrm{Z}$ & $\mathrm{C}$ \\
\hline Proton pump inhibitors & 2 & & 1 & 2 & 1 & & 4 & 2 \\
\hline Non-steroidal anti-inflammatory drugs & 1 & 1 & 2 & 2 & & & 3 & 3 \\
\hline Antispasmodics and other drugs Altering gut motility & & & 1 & 4 & & & 1 & 4 \\
\hline Expectorant and demulcent Cough preparations & & & 2 & & 3 & & 5 & 0 \\
\hline Benzylpenicillin and phenoxymethylpenicillin & & 2 & 1 & 1 & 1 & & 2 & 3 \\
\hline Combined hormonal contraceptives & & & 1 & 4 & & & 1 & 4 \\
\hline Antibacterials & 1 & & 1 & 1 & 2 & & 4 & 1 \\
\hline
\end{tabular}

Table 3: Maternal co-medication. All maternal medications received from pregnancy onset to last antiviral medication treatment or comparator date plus 28 days, reported by trimester of treatment initiation among women with a pregnancy outcome between $1^{\text {st }}$ December 2009 and $30^{\text {th }}$ November 2010. (Excerpted to include BNF categories dispensed to at least five cohort members).

\section{Maternal and Infant Outcomes}

There were 64 zanamivir-exposed and 68 comparator pregnancies in which the primary identification occurred through the GP records of the pregnancy (as opposed to having come from birth records). All pregnancies resulted in live birth (Table 4). Prematurity and low birth weight information was available for only a few records (Table 4). Maternal outcomes consistent with manifestations of ILI were in excess in the zanamivir group. All of the outcomes that were in excess in the zanamivir-prescribed pregnancies were consistent with an acute viral episode (Table 4).

\begin{tabular}{|c|c|c|c|c|c|c|c|c|c|}
\hline \multirow[b]{3}{*}{ 4a. Maternal outcome diagnoses } & & \multicolumn{6}{|c|}{ Trimester } & & \\
\hline & & \multicolumn{2}{|c|}{$\# 1$} & \multicolumn{2}{|c|}{$\# 2$} & \multicolumn{2}{|c|}{$\# 3$} & \multicolumn{2}{|c|}{ Total } \\
\hline & & Z & C & Z & $\mathrm{C}$ & $\mathrm{Z}$ & $\mathrm{C}$ & $\mathrm{Z}$ & $\mathrm{C}$ \\
\hline & $\mathrm{n}$ & 44 & 41 & 88 & 91 & 12 & 12 & 144 & 144 \\
\hline Flu like illness & & 11 & & 16 & & 1 & & 28 & 0 \\
\hline Influenza & & 8 & & 9 & & 2 & & 19 & 0 \\
\hline $\mathrm{C} / \mathrm{O}$ cough & & 2 & & 9 & & 3 & & 14 & 0 \\
\hline Influenza-like symptoms & & 7 & & 6 & & & & 13 & 0 \\
\hline Feels hot/feverish & & 2 & & 6 & 1 & & & 8 & 1 \\
\hline Influenza A (H1N1) swine flu & & 4 & & 2 & & 3 & & 9 & 0 \\
\hline $\mathrm{C} / \mathrm{O}$ headache & & 2 & & 4 & 2 & & & 6 & 2 \\
\hline Sore throat symptom & & 4 & & 4 & & & & 8 & 0 \\
\hline Nose running & & 2 & & 3 & 1 & & & 5 & 1 \\
\hline Aching muscles & & 3 & & 2 & 1 & & & 5 & 1 \\
\hline Cough & & & & 4 & 1 & & & 4 & 1 \\
\hline Patient pregnant & & 1 & 4 & & & & & 1 & 4 \\
\hline Abdominal pain & & 2 & & 1 & & 1 & & 4 & 0 \\
\hline Fever symptoms & & 1 & & 3 & & & & 4 & 0 \\
\hline Sneezing symptoms & & & & 3 & 1 & & & 3 & 1 \\
\hline Low back pain & & & & 2 & 1 & & 1 & 2 & 2 \\
\hline Tiredness symptom & & 1 & & 2 & & & & 3 & 0 \\
\hline General symptom NOS & & 1 & & 2 & & & & 3 & 0 \\
\hline Contact - infectious disease & & 1 & & 2 & & & & 3 & 0 \\
\hline Tired all the time & & & 1 & & 1 & & & 0 & 2 \\
\hline Shortness of breath & & & & 2 & & & & 2 & 0 \\
\hline Feels unwell & & 1 & & 1 & & & & 2 & 0 \\
\hline $\mathrm{C} / \mathrm{O}$ a pain & & 2 & & & & & & 2 & 0 \\
\hline
\end{tabular}




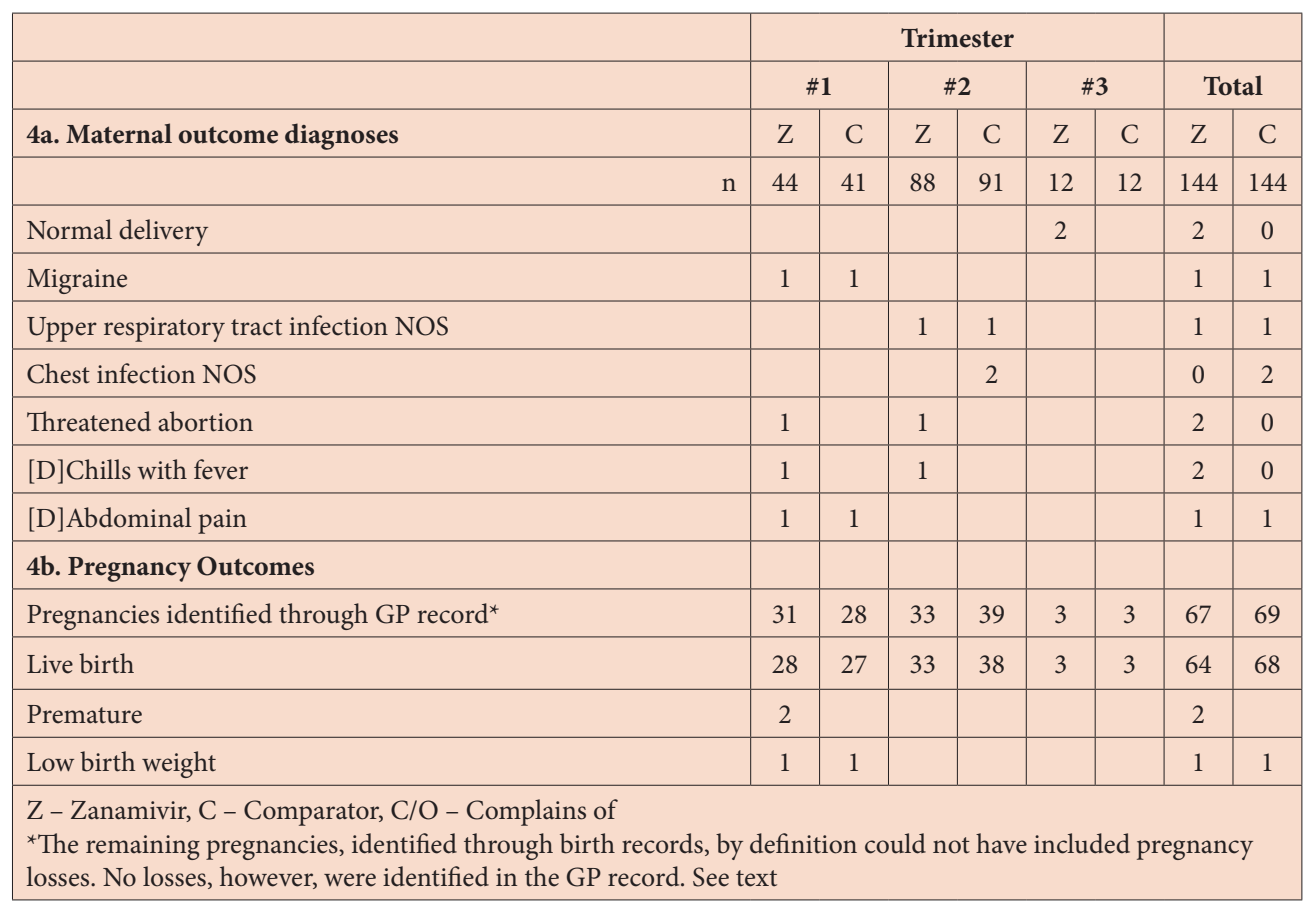

Table 4: Maternal outcome diagnoses (4a) and pregnancy outcomes (4b). Maternal diagnoses appearing for the first time following cohort entry date (zanamivir initiation or comparator date) and within 28 days following last zanamivir medication prescribing or comparator date, reported by trimester of treatment initiation, among women with a pregnancy outcome between $1^{\text {st }}$ December 2009 and $30^{\text {th }}$ November 2010.

The 28 days following birth were examined in the GP record for all types of diagnoses. Table 5 presents diagnoses relating to possible congenital anomalies ( $\mathrm{n}=24$ zanamivir group, $\mathrm{n}=26$ comparator group). The number of major congenital anomalies reported among comparators (14/144) was higher than among the zanamivir-exposed group (4/144). The greatest imbalance was in cardiac defects, consisting of atrial septal defect, ventricular septal defect and patent ductus arteriosus (collectively, one in zanamivir group, six in comparators).

\begin{tabular}{|c|c|c|c|c|c|c|c|c|c|}
\hline \multirow{3}{*}{ EUROCAT Major Congenital Anomalies¥ } & \multirow[b]{3}{*}{ EUROCAT Subgroup } & \multicolumn{8}{|c|}{ Trimester } \\
\hline & & \multicolumn{2}{|c|}{$\# 1$} & \multicolumn{2}{|c|}{$\# 2$} & \multicolumn{2}{|c|}{ \#3 } & \multicolumn{2}{|c|}{ Total } \\
\hline & & $\mathrm{Z}$ & $\mathrm{C}$ & $\mathrm{Z}$ & $\mathrm{C}$ & $\mathrm{Z}$ & $\mathrm{C}$ & $\mathrm{Z}$ & $\mathrm{C}$ \\
\hline Tracheo-esophageal fistula & Digestive & & & & & & 1 & 0 & 1 \\
\hline Atresia of duodenum & Digestive & & & & & & 1 & 0 & 1 \\
\hline Congenital optic disc coloboma & Eye & & & & & & 1 & 0 & 1 \\
\hline Ventricular septal defect & Cardiac & & & 1 & & & 1 & 1 & 1 \\
\hline Atrial septal defect NOS & Cardiac & & & & 1 & & 2 & 0 & 3 \\
\hline Patent ductus arteriosus at 37 weeks or later & Cardiac & & & & 1 & & 1 & 0 & 2 \\
\hline Choanal atresia, unspecified & Respiratory & & & & & & 1 & 0 & 1 \\
\hline Congenital cystic adenomatoid malformation of the lung & Respiratory & 1 & & & & & & 1 & 0 \\
\hline Congenital flexion contracture of hip & Limb & & 1 & & & & & 0 & 1 \\
\hline Developmental dysplasia of the hip & Limb & 1 & & 1 & 1 & & & 2 & 1 \\
\hline Skull or face bone anomaly NOS & Musculo-skeletal & & & & & & 1 & 0 & 1 \\
\hline Osteogenesis imperfecta & Musculo-skeletal & & 1 & & & & & 0 & 1 \\
\hline TOTAL MAJOR $\ddagger$ & & 2 & 2 & 2 & 3 & 0 & 9 & 4 & 14 \\
\hline
\end{tabular}




\begin{tabular}{|c|c|c|c|c|c|c|c|c|c|}
\hline \multirow{3}{*}{ EUROCAT Minor Anomalies $¥$} & \multirow[b]{3}{*}{ EUROCAT Subgroup } & \multicolumn{8}{|c|}{ Trimester } \\
\hline & & \multicolumn{2}{|c|}{$\# 1$} & \multicolumn{2}{|c|}{$\# 2$} & \multicolumn{2}{|c|}{$\# 3$} & \multicolumn{2}{|c|}{ Total } \\
\hline & & $\mathrm{Z}$ & $\mathrm{C}$ & $\mathrm{Z}$ & $\mathrm{C}$ & $\mathrm{Z}$ & $\mathrm{C}$ & $\mathrm{Z}$ & $\mathrm{C}$ \\
\hline Laryngomalacia & Pulmonary & & & & & & 1 & 0 & 1 \\
\hline Right inguinal hernia & Gastro-intestine & & 1 & & & & & 0 & 1 \\
\hline Inguinal hernia & Gastro-intestine & & 1 & 1 & & & & 1 & 1 \\
\hline Umbilical hernia & Gastro-intestine & 1 & & 1 & & & & 2 & 0 \\
\hline Hydrocele & Genitalia & & & 1 & & & & 1 & 0 \\
\hline Tight foreskin & Genitalia & 1 & & & & & & 1 & 0 \\
\hline Phimosis & Genitalia & & & & 1 & & & 0 & 1 \\
\hline Retractile testis & Genitalia & 1 & & & & & & 1 & 0 \\
\hline Clicking hip & Limb & & & & 1 & & & 0 & 1 \\
\hline Clicking knee & Limb & 1 & & & & & & 1 & 0 \\
\hline Talipes, unspecified & Limb & 1 & & & & & & 1 & 0 \\
\hline Congenital positional talipes & Limb & & & 2 & & & & 2 & 0 \\
\hline Tongue tie - ankyloglossia & Head & & 1 & & & 1 & & 1 & 1 \\
\hline Tongue tie & Head & & & 1 & & & & 1 & 0 \\
\hline Plagiocephaly & Head & & & & 1 & & & 0 & 1 \\
\hline Torticollis - symptom & Neck & & & 1 & & & & 1 & 0 \\
\hline Strawberry nevus & Skin & & & & 1 & & & 0 & 1 \\
\hline Mongolian blue spot & Skin & & & & 1 & & & 0 & 1 \\
\hline [D]Heart murmur, functional & Cardiac & & & 1 & & & & 1 & 0 \\
\hline [D] heart murmur, innocent & Cardiac & & 1 & & & & & 0 & 1 \\
\hline \multirow[t]{2}{*}{ TOTAL MINOR $\ddagger$} & & 5 & 4 & 8 & 5 & 1 & 1 & 14 & 10 \\
\hline & & \multicolumn{8}{|c|}{ Trimester } \\
\hline \multirow[t]{2}{*}{ Conditions not EUROCAT congenital anomalies } & & \multicolumn{2}{|c|}{$\# 1$} & \multicolumn{2}{|c|}{$\# 2$} & \multicolumn{2}{|c|}{$\# 3$} & \multicolumn{2}{|c|}{ Total } \\
\hline & EUROCAT Subgroup & $\mathrm{Z}$ & $\mathrm{C}$ & $\mathrm{Z}$ & $\mathrm{C}$ & $\mathrm{Z}$ & $\mathrm{C}$ & $\mathrm{Z}$ & $\mathrm{C}$ \\
\hline Hypoglycemia unspecified & $\mathrm{N} / \mathrm{A}$ & & & & & 1 & & 1 & 0 \\
\hline Achalasia of cardia & N/A & & & 1 & & & & 1 & 0 \\
\hline Anal fissure & N/A & & & 1 & & & & 1 & 0 \\
\hline Penile disorders NOS & N/A & & & 1 & & & 1 & 1 & 1 \\
\hline Calcaneal spur & N/A & & & 1 & & & & 1 & 0 \\
\hline Erb's palsy & N/A & & & & & 1 & & 1 & 0 \\
\hline Meconium obstruction NOS & N/A & & & & 1 & & & 0 & 1 \\
\hline TOTAL OTHERS & & 0 & 0 & 4 & 1 & 2 & 1 & 6 & 2 \\
\hline $\begin{array}{l}\text { Z - Zanamivir, C - Comparator } \\
\ddagger-\text { Totals represent total number of malformations } \\
¥-\text { The EUROCAT guide and reference document }\end{array}$ & ; there could be multip & & & per in & & & & & \\
\hline
\end{tabular}

Table 5: Major, minor and non-EUROCAT congenital anomalies. Diagnoses observed in zanamivir and comparator groups among women with a pregnancy outcome between $1^{\text {st }}$ December 2009 and $30^{\text {th }}$ November 2010.

Among women with first trimester exposure to zanamivir and their comparators, there were two major congenital anomalies in the zanamivir group and two in the comparator group. There were two major congenital anomalies in the exposed cohort and three in the comparator group among the second trimester exposures, while among those exposed in the third trimester there were nine documented major congenital anomalies in the comparator group and none in the exposed cohort.

\section{Discussion}

This comparison of 144 zanamivir-exposed pregnancies to a like number showed no evidence for an increased risk of major congenital malformations associated with zanamivir use, and no evidence for an increased risk of poor pregnancy or maternal outcomes. 
Because this was a retrospective study based on general practices, the compared cohorts consisted essentially of pregnant women prescribed zanamivir and concurrently pregnant women with no prescription. In neither group was influenza either formally demonstrated or ruled out. Although there was abundant recording of diagnoses and symptoms consistent with both influenza and other ILI in the zanamivir recipients (and basically none in the comparators), there was no documentation that influenza was assessed by any laboratory procedure in the great majority of patients.

It is possible that some of the exposures were misclassified, as prescription may not always guarantee use of a drug. Moreover it is possible that some of the comparator pregnancies were in fact exposed to the drug, prescribed outside of the general practice. The number is likely to be very small. Additionally, there was a lack of access to pregnant women with ILI who were not given zanamivir. Zanamivir use is uncommon in pregnant women in general, and the listings of reasons for encounters in the comparison women showed an almost complete absence both of influenza diagnosis and of symptoms that would be compatible with ILI.

Covariate control was not part of the planned data analysis due to the exploratory nature of this study, the restriction to group reports, and the relatively small number of zanamivir-prescribed cohort members.

The absence of records of pregnancy losses was a noteworthy limitation in this study, as only live births were documented in the GP record. This may be due, in part, to the small numbers in the cohort and among them the small number of individuals with any note of antenatal care in the GP record. Antenatal care in the UK is carried out in specialized clinics. Often these are in the general practice to which a woman belongs, and in principle the notes of care flow into the GP record. In fact, a majority of the zanamivir-exposed pregnancies were identified only from a record of live birth and not from a record of pregnancy, demonstrating that pregnancy and pregnancy outcome data were frequently missing from the GP record.

This study demonstrated that infants born from zanamivir-exposed pregnancies could be usefully compared to infants from other pregnancies in GP data from the UK. The late cohort entry (second or third trimester) of the majority of women accounts for a diminished expectation of pregnancy loss in the cohort, by comparison to what one would anticipate in a cohort fully identified in the first trimester.

\section{Conclusion}

Zanamivir-exposed pregnant women have a wide range of symptoms of and treatments for ILI that complicate comparisons with untreated women. In our study, there was no evidence of zanamivir-associated risks in the mother or to the pregnancy. Congenital anomalies occurred in similar proportions in zanamivir exposed and comparator fetuses.

\section{Acknowledgement}

GlaxoSmithKline provided funding for this study. All listed authors meet the criteria for authorship set forth by the International Committee for Medical Journal Editors. The authors thank Fiona Hill of Cegedim Strategic Data Medical Research, Ltd. for her help with the methodology employed to identify pregnancies and outcomes.

\section{References}

1. Dodds L, McNeil SA, Fell DB, Allen VM, Coombs A, et al. (2007) Impact of influenza exposure on rates of hospital admissions and physician visits because of respiratory illness among pregnant women. CMAJ 176: 463-8.

2. Fiore AE, Shay DK, Broder K, Iskander JK, Uyeki TM, et al. (2008) Prevention and control of influenza: recommendations of the Advisory Committee on Immunization Practices (ACIP), 2008. MMWR Recomm Rep 57: 1-60.

3. Dawood FS, Iuliano AD, Reed C, Meltzer MI, Shay DK, et al. (2012) Estimated global mortality associated with the first 12 months of 2009 pandemic influenza A H1N1 virus circulation: a modelling study. Lancet Infect Dis 12: 687-95.

4. McLean E, Pebody R (2010) Epidemiological report of pandemic (H1N1) 2009 in the UK: April 2009-May 2010.

5. Pebody RG, McLean E, Zhao H, Cleary P, Bracebridge S, et al. (2010) Pandemic Influenza A (H1N1) 2009 and mortality in the United Kingdom: risk factors for death, April 2009 to March 2010. Euro Surveill 20: 19571.

6. Creanga AA, Kamimoto L, Newsome K, D'Mello T, Jamieson DJ, et al. (2011) Seasonal and 2009 pandemic influenza A (H1N1) virus infection during pregnancy: a population-based study of hospitalized cases. Am J Obstet Gynecol 204: S38-45.

7. Creanga AA, Johnson TF, Graitcer SB, Hartman LK, Al-Samarrai T, et al. (2010) Severity of 2009 pandemic influenza A (H1N1) virus infection in pregnant women. Obstet Gynecol 115: 717-26.

8. Siston AM, Rasmussen SA, Honein MA, Fry AM, Seib K, et al. (2010) Pandemic 2009 influenza A(H1N1) virus illness among pregnant women in the United States. JAMA 303: 1517-25.

9. Mosby LG, Rasmussen SA, Jamieson DJ (2011) 2009 pandemic influenza A (H1N1) in pregnancy: a systematic review of the literature. Am J Obstet Gynecol 205: $10-8$.

10. Centers for Disease Control (CDC) (2011) Maternal and infant outcomes among severely ill pregnant and postpartum women with 2009 pandemic influenza A (H1N1)--United States, April 2009-August 2010. MMWR Morb Mortal Wkly Rep 60: 1193-6.

11. Yates L, Pierce M, Stephens S, Mill AC, Spark P, et al. (2010) Influenza A/H1Nlv in pregnancy: an investigation of the characteristics and management of affected women and the relationship to pregnancy outcomes for mother and infant. Health Technol Assess 14: 109-82.

12. Donner B, Niranjan V, Hoffmann G (2010) Safety of oseltamivir in pregnancy: a review of preclinical and clinical data. Drug Saf 33: 631-42. 
13. Acs N, Banhidy F, Puho E, Czeizel AE (2005) Maternal influenza during pregnancy and risk of congenital abnormalities in offspring. Birth Defects Res A Clin Mol Teratol 73: 989-96.

14. Tanaka T, Nakajima K, Murashima A, Garcia-Bournissen F, Koren G, et al. (2009) Safety of neuraminidase inhibitors against novel influenza A (H1N1) in pregnant and breastfeeding women. CMAJ 181: 55-8.

15. Beck S, Wojdyla D, Say L, Betran AP, Merialdi M, et al. (2010) The worldwide incidence of preterm birth: a systematic review of maternal mortality and morbidity. Bull World Health Organ 88: 31-8.

16. ANZIC Influenza Investigators and Australasian Maternity Outcomes Surveillance System (2010) Critical illness due to 2009 A/H1N1 influenza in pregnant and postpartum women: population based cohort study. BMJ 340: c1279.

17. Gerardin P, El AR, Cyrille B, Gabriele M, Guillermin P, et al. (2010) Low clinical burden of 2009 pandemic influenza A (H1N1) infection during pregnancy on the island of La Reunion. PLoS One 5: e10896.

18. Honarvar B, Asadi N, Ghaffarpasand F, Moghadami M, Kasraeian M (2010) Pregnancy outcomes among patients infected with pandemic H1N1 influenza virus in Shiraz, Iran. Int J Gynaecol Obstet 111: 86-7.

19. Jamieson DJ, Honein MA, Rasmussen SA, Williams JL, Swerdlow DL, et al. (2009) H1N1 2009 influenza virus infection during pregnancy in the USA. Lancet 374: 451-8.

20. Louie JK, Acosta M, Jamieson DJ, Honein MA (2010) Severe 2009 H1N1 influenza in pregnant and postpartum women in California. N Engl J Med 362: 27-35. 21. Rasmussen SA, Kissin DM, Yeung LF, Macfarlane K, Chu SY, et al. (2011) Preparing for influenza after 2009 H1N1: special considerations for pregnant women and newborns. Am J Obstet Gynecol 204: S13-20.

22. Rasmussen SA, Jamieson DJ (2012) Influenza and pregnancy in the United States: before, during, and after 2009 H1N1. Clin Obstet Gynecol 55: 487-97.

23. Glaxo Wellcome Incorporated (2009) Relenza (zanamivir for inhalation).

24. Freund B, Gravenstein S, Elliott M, Miller I (1999) Zanamivir: a review of clinical safety. Drug Saf 21: 267-81.

25. Svensson T, Granath F, Stephansson O, Kieler H (2011) Birth outcomes among women exposed to neuraminidase inhibitors during pregnancy. Pharmacoepidemiol Drug Saf 20: 1030-4.

26. European Medicines Agency (EMA) CfMPfHU (2009) Novel influenza (H1N1) outbreak: Tamiflu (oseltamivir), Relenza (zanamivir).

27. UK National Health Service (NHS) Information Authority (2013) Clinical Terminology Browser v1.04. Available as "Clinical Terminology Browser" from the UK National Health Service Technology Data Reference Update Distribution.

28. European Surveillance of Congenital Anomalies (EUROCAT) (2005) EUROCAT guide and reference documents, Instructions for the Registration and Surveillance of Congenital Anomalies. 